02

\title{
Спектроскопическое и квантово-химическое исследование галогенсодержащих производных поли-N-эпоксипроилкарбазола
}

\author{
(C) Е.В. Селиверстова, Н.Х. Ибраев, А.К. Нурмаханова, Д.А. Темирбаева \\ Институт молекулярной нанофотоники, Карагандинский государственный университет им. Е.А. Букетова, \\ 100028 Караганда, Казахстан \\ e-mail: genia_sv@mail.ru, niazibrayev@mail.ru \\ Поступила в Редакцию 16.03.2018 г.
}

Приведены результаты исследования оптических свойств проводящих полимеров - производных РЕРС с тяжелыми атомами. Показано, что тяжелый атом в структуре полимерной молекулы приводит к батохромному сдвигу спектров поглощения, флуоресценции и фосфоресценции. Это является следствием понижения энергии электронных уровней из-за изменения распределения электронной плотности в системе $\pi$-электронов в хромофорах 2IPEРС и 3ВrРЕРС. В спектрах флуоресценции РЕРС с тяжелыми атомами, как и в материнском полимере, можно выделить две полосы. Полоса излучения с максимумом на $380 \mathrm{~nm}$ принадлежит мономерным центрам свечения, а длинноволновая с максимумом около $420 \mathrm{~nm}$ - эксимерам полимеров. Тяжелый атом изменяет соотношение интенсивностей мономерной и эксимерной полос. Времена жизни флуоресценции в присутствии тяжелого атома также уменьшаются. Квантово-химическая оценка констант внутримолекулярных переходов показала, что в галогенсодержащих РЕРС вероятность синглеттриплетной интеркомбинационной конверсии выше по сравнению с РЕРС. Это приводит к заметно более интенсивной фосфоресценции йод- и бромсодержащих полимеров и сокращению времени жизни свечения. Полученные результаты могут быть использованы при разработке композитных материалов на основе фотопроводящих полимеров для фотоэнергетики и оптоэлектроники.

DOI: $10.21883 /$ OS.2018.10.46698.74-18

\section{Введение}

Полупроводниковые полимеры активно используются как для изготовления устройств молекулярной электроники в качестве проводящих элементов [1], так и в устройствах хранения информации [2]. В последнее время наиболее популярной областью использования таких полимеров является органическая фотовольтаика [3-6].

В работах $[7,8]$ установлено, что в пленках поли-Nэпоксипропилкарбазола (РЕРС), допированных катионным полиметиновым красителем, дополнительным каналом релаксации возбужденных состояний красителя является образование и рекомбинация электрондырочных пар (ЭДП), что проявляется в появлении рекомбинационной люминесценции. Было показано, что добавление примеси КІ в полимерную пленку приводит к снижению величины магнитного эффекта на рекомбинационную люминесценцию вследствие конкуренции между синглетным и триплетным каналами образования ЭДП. В солнечных ячейках полимерная матрица наряду с участием в генерации и рекомбинации носителей заряда через невозбужденное основное состояние может поглощать солнечное излучение в УФ области спектра. Поэтому важными становятся вопросы трансформации световой энергии через возбужденные электронные состояния используемых материалов.

В настоящей работе приведены результаты исследования возбужденных синглетных и триплетных состояний фотопроводящих полимеров - производных РЕРС с тяжелыми атомами. Интерес к галогенпроизводным РЕРС связан с тем, что тяжелые атомы в полимерном соединении могут усиливать спин-орбитальное взаимодействие как внутри полимера, так и в примесных молекулах красителя [9]. Это позволит использовать долгоживущие возбужденные триплетные состояния молекул красителя для увеличения вероятности образования ЭДП. Рост числа ЭДП приведет к увеличению фототока в полимерных солнечных ячейках.

\section{Экспериментальная часть}

Для исследования были выбраны РЕРС и его производные, модифицированные тяжелыми атомами дийод-РЕРС (2IРЕРС) и трибром-РЕРС (3ВrРЕРС) (рис. 1).

Пленки были приготовлены методом полива из растворов полимеров в хлороформе. Концентрация полимера в пленке была равна 7 mass\%. Пленки наносили на подложки из плавленного кварца. Хлороформ марки х.ч. дополнительно очищали методом перегонки.

Спектры поглощения исследуемых полимеров были измерены на спектрофотометре Cary-300 (Agilent). Спектры флуоресценции и фосфоресценции были измерены на спектрофлуориметре Cary Eclipse (Agilent). Времена жизни возбужденного состояния полимеров измерялись с помощью импульсного спектрофлуориметра с пикосекундным возбуждением с регистрацией в режиме время-коррелированного счета фотонов (Becker\&Hikl, Germany). Для возбуждения образцов был использован лазер с $\lambda_{\mathrm{ex}}=375 \mathrm{~nm}$. 
<smiles>CCCC(CC)OC(CC)Cn1c2ccccc2c2cc(I)ccc21</smiles><smiles>CCC(CCn1c2ccc(Br)cc2c2cc(Br)c(Br)cc21)OO</smiles>

3BrPEPC

Рис. 1. Структурные формулы полимеров.

Поиск оптимальной конформации осуществляли с помощью методов молекулярной механики, а расчет спектральных характеристик - неэмпирическим методом TD DFT/B3LYP с использованием программного пакета Gaussian 09W Revision-A.02. Расчет проводился для мономерных звеньев исследуемых полимеров в вакууме. Оценка скоростей фотофизических процессов проводилась полуэмприческим методом INDO/s со спектроскопической параметризацией согласно работам $[10,11]$.

\section{Результаты и обсуждение}

Моделирование электронной структуры и фотофизических процессов в молекулах РЕРС, 2IPЕРС и $3 \mathrm{BrPEPC}$ было выполнено для мономерных звеньев с конформацией, установленной методом молекулярной механики. Расчет показал, что наиболее оптимальной и вероятной конформацией мономерного звена исследуемых полимеров является такая, в которой карбазольный цикл выведен из плоскости молекулы и повернут на $9.5^{\circ}$ против часовой стрелки. При этом угол наклона между данным фрагментом и эпоксипропиловым остатком равен $114^{\circ}$.

Оценка величины заряда на атомах мономерных звеньев РЕРС и его галогенпроизводных по Малликену в мономерных звеньях показала, что положительный заряд сконцентрирован на центральном пиррольном кольце карбазола. Атомы углерода в фенильных циклах заряжены отрицательно, а соседние атомы водорода имеют положительный заряд. Отрицательный заряд расположен на атоме кислорода и двух атомах углерода, при помощи которых мономерные звенья соединяются между собой и образуют полимерное звено. При возбуждении молекулы заряды на атомах углерода, входящих в фенольные кольца, уменьшаются. В то же время отрицательный
Таблица 1. Рассчитанные спектральные характеристики мономерного звена РЕРС и его производных

\begin{tabular}{|c|c|c|c|c|c|c|}
\hline $\begin{array}{c}\text { Состоя- } \\
\text { ние }\end{array}$ & $E, \mathrm{eV}$ & $\lambda, \mathrm{nm}$ & $f$ & $\begin{array}{c}\text { Занятая } \\
\text { молеку- } \\
\text { лярная } \\
\text { орбиталь }\end{array}$ & $\begin{array}{c}\text { Незанятая } \\
\text { молеку- } \\
\text { лярная } \\
\text { орбиталь }\end{array}$ & $\begin{array}{c}\% \\
\text { вклада }\end{array}$ \\
\hline \multicolumn{7}{|c|}{ PEPC } \\
\hline$S_{1}$ & 3.6886 & 336 & 0.05 & $\begin{array}{l}\text { HOMO } \\
\text { HOMO-1 }\end{array}$ & $\begin{array}{l}\text { LUMO } \\
\text { LUMO+1 }\end{array}$ & $\begin{array}{l}69 \\
14\end{array}$ \\
\hline$S_{2}$ & 4.2739 & 290 & 0.30 & $\begin{array}{l}\text { HOMO } \\
\text { HOMO-1 } \\
\text { HOMO-1 }\end{array}$ & $\begin{array}{l}\text { LUMO } \\
\text { LUMO+1 } \\
\text { LUMO }\end{array}$ & $\begin{array}{l}63 \\
20 \\
11\end{array}$ \\
\hline$S_{3}$ & 4.9778 & 249 & 0.45 & $\begin{array}{l}\text { HOMO } \\
\text { HOMO-1 } \\
\text { HOMO }\end{array}$ & $\begin{array}{l}\text { LUMO+1 } \\
\text { LUMO } \\
\text { LUMO+2 }\end{array}$ & $\begin{array}{l}57 \\
26 \\
16\end{array}$ \\
\hline$S_{4}$ & 5.1023 & 243 & 0.06 & $\begin{array}{l}\text { HOMO-1 } \\
\text { HOMO-2 } \\
\text { HOMO-1 }\end{array}$ & $\begin{array}{l}\text { LUMO } \\
\text { LUMO } \\
\text { LUMO+2 }\end{array}$ & $\begin{array}{l}17 \\
62 \\
18\end{array}$ \\
\hline$S_{5}$ & 5.1425 & 241 & 0.02 & $\begin{array}{l}\text { HOMO-1 } \\
\text { HOMO }\end{array}$ & $\begin{array}{l}\text { LUMO+1 } \\
\text { LUMO+2 }\end{array}$ & $\begin{array}{l}57 \\
36\end{array}$ \\
\hline$S_{6}$ & 5.5936 & 221 & 0.12 & $\begin{array}{l}\text { HOMO } \\
\text { HOMO-3 }\end{array}$ & $\begin{array}{l}\text { LUMO+2 } \\
\text { LUMO }\end{array}$ & $\begin{array}{l}53 \\
31\end{array}$ \\
\hline$S_{7}$ & 5.6936 & 218 & 0.51 & $\begin{array}{l}\text { HOMO } \\
\text { HOMO } \\
\text { HOMO-1 }\end{array}$ & $\begin{array}{l}\text { LUMO+3 } \\
\text { LUMO+1 } \\
\text { LUMO }\end{array}$ & $\begin{array}{l}60 \\
19 \\
18\end{array}$ \\
\hline$S_{8}$ & 5.9438 & 209 & 0.11 & $\begin{array}{l}\text { HOMO-2 } \\
\text { HOMO } \\
\text { HOMO-1 }\end{array}$ & $\begin{array}{l}\text { LUMO } \\
\text { LUMO+3 } \\
\text { LUMO+2 }\end{array}$ & $\begin{array}{l}44 \\
41 \\
15\end{array}$ \\
\hline$S_{9}$ & 6.0158 & 206 & 0.05 & $\begin{array}{l}\text { HOMO-1 } \\
\text { HOMO-3 } \\
\text { HOMO }\end{array}$ & $\begin{array}{l}\text { LUMO+2 } \\
\text { LUMO } \\
\text { LUMO+3 }\end{array}$ & $\begin{array}{l}53 \\
20 \\
21\end{array}$ \\
\hline$S_{10}$ & 6.0964 & 203 & 0.02 & $\begin{array}{l}\text { HOMO-3 } \\
\text { HOMO-2 } \\
\text { HOMO-1 }\end{array}$ & $\begin{array}{l}\text { LUMO } \\
\text { LUMO } \\
\text { LUMO+2 }\end{array}$ & $\begin{array}{l}60 \\
18 \\
18 \\
\end{array}$ \\
\hline \multicolumn{7}{|c|}{ 2IPEPC } \\
\hline$S_{1}$ & 3.4364 & 361 & 0.025 & $\begin{array}{l}\text { HOMO } \\
\text { HOMO-1 }\end{array}$ & $\begin{array}{l}\text { LUMO } \\
\text { LUMO+3 }\end{array}$ & $\begin{array}{l}69 \\
10\end{array}$ \\
\hline$S_{2}$ & 4.0127 & 309 & 0.08 & $\begin{array}{l}\text { HOMO-1 } \\
\text { HOMO } \\
\text { HOMO-2 }\end{array}$ & $\begin{array}{l}\text { LUMO } \\
\text { LUMO+3 } \\
\text { LUMO }\end{array}$ & $\begin{array}{l}64 \\
25 \\
12\end{array}$ \\
\hline$S_{3}$ & 4.4820 & 276 & 0.11 & $\begin{array}{l}\text { HOMO } \\
\text { HOMO-2 }\end{array}$ & $\begin{array}{l}\text { LUMO+2 } \\
\text { LUMO }\end{array}$ & $\begin{array}{l}51 \\
45\end{array}$ \\
\hline$S_{4}$ & 4.7228 & 262 & 0.001 & $\begin{array}{l}\text { HOMO-1 } \\
\text { HOMO }\end{array}$ & $\begin{array}{l}\text { LUMO+2 } \\
\text { LUMO+1 }\end{array}$ & $\begin{array}{l}65 \\
17\end{array}$ \\
\hline$S_{5}$ & 4.7780 & 259 & 0.74 & $\begin{array}{l}\text { HOMO-2 } \\
\text { HOMO }\end{array}$ & \begin{tabular}{|l|} 
LUMO \\
LUMO+3 \\
\end{tabular} & $\begin{array}{l}51 \\
38 \\
\end{array}$ \\
\hline \multirow[b]{2}{*}{$S_{1}$} & & & $3 \mathrm{BrP}$ & EPC & & \\
\hline & 3.4072 & 364 & 0.03 & $\begin{array}{l}\text { HOMO } \\
\text { HOMO-1 }\end{array}$ & $\begin{array}{l}\text { LUMO } \\
\text { LUMO+2 }\end{array}$ & $\begin{array}{l}70 \\
12\end{array}$ \\
\hline$S_{2}$ & 3.9947 & 310 & 0.007 & $\begin{array}{l}\text { HOMO-1 } \\
\text { HOMO }\end{array}$ & $\begin{array}{l}\text { LUMO } \\
\text { LUMO+3 }\end{array}$ & $\begin{array}{l}62 \\
28\end{array}$ \\
\hline$S_{3}$ & 4.5800 & 271 & 0.16 & $\begin{array}{l}\text { HOMO-2 } \\
\text { HOMO-1 }\end{array}$ & $\begin{array}{l}\text { LUMO } \\
\text { LUMO }\end{array}$ & $\begin{array}{l}68 \\
12\end{array}$ \\
\hline$S_{4}$ & 4.6015 & 269 & 0.35 & $\begin{array}{l}\text { HOMO } \\
\text { HOMO-3 }\end{array}$ & $\begin{array}{l}\text { LUMO+3 } \\
\text { LUMO+2 }\end{array}$ & $\begin{array}{l}55 \\
33\end{array}$ \\
\hline$S_{5}$ & 4.7169 & 262 & 0.40 & $\begin{array}{l}\text { HOMO-3 } \\
\text { HOMO-1 }\end{array}$ & $\begin{array}{l}\text { LUMO+2 } \\
\text { LUMO+2 }\end{array}$ & $\begin{array}{l}57 \\
27\end{array}$ \\
\hline
\end{tabular}




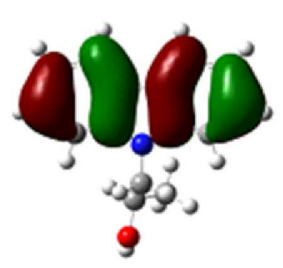

HOMO - 1 PEPC

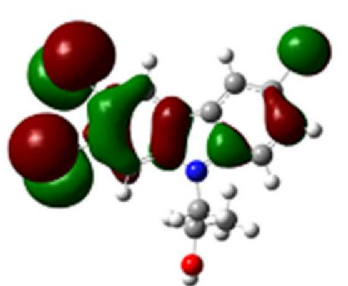

HOMO - 13 BrPEPC

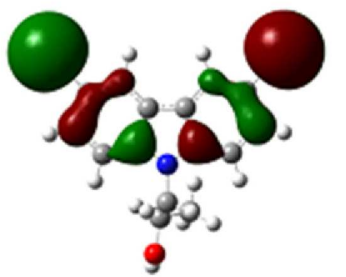

HOMO - 1 2IPEPC

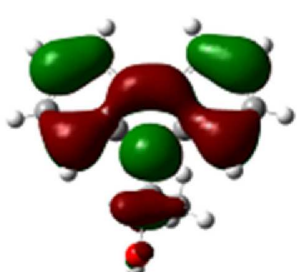

HOMO PEPC

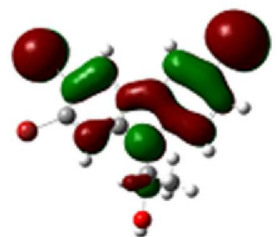

HOMO 3BrPEPC

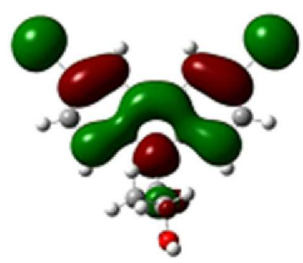

HOMO 2IPEPC

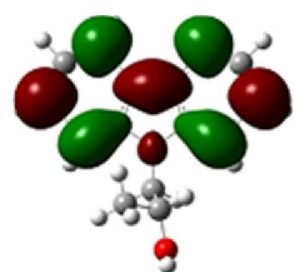

LUMO PEPC

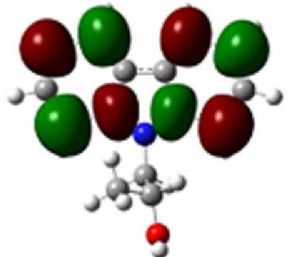

LUMO + 1 PEPC
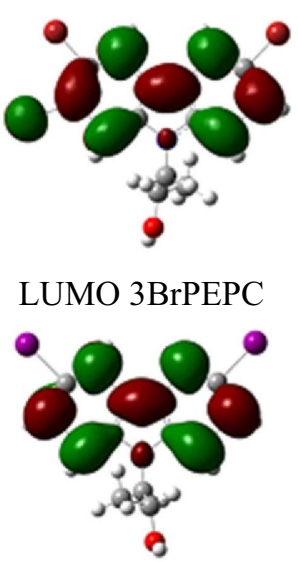

LUMO 2IPEPC

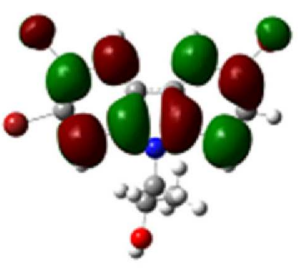

LUMO + 1 3BrPEPC

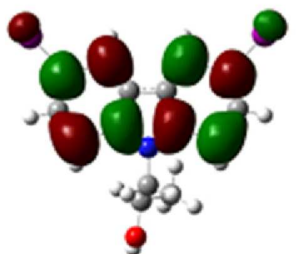

$\mathrm{LUMO}+1$ 2IPEPC

Рис. 2. Распределение электронной плотности на молекулярных орбиталях РЕРC, 2IPEPC и 3BrPEPC.

заряд на атоме азота пиридинового цикла и атомах углерода увеличивается. Это приводит к росту заселенности данных связей и свидетельствует о возможности осуществления внутримолекулярного переноса заряда. Для молекул с тяжелыми атомами были получены аналогичные результаты. Таким образом, наличие тяжелого атома не оказывает заметного влияния на распределение зарядов внутри изучаемых соединений.

При расчете электронной структуры молекулы РЕРС был получен спектр поглощения с максимумами на 249, 290 и $336 \mathrm{~nm}$. Силы осцилляторов равны 0.45, 0.3 и 0.05 соответственно. Первая полоса поглощения обусловлена переходом молекулы из состояния $S_{0}$ в $S_{3}$, а следующие полосы переходами $S_{0} \rightarrow S_{2}$ и $S_{0} \rightarrow S_{1}$. Переход $S_{0} \rightarrow S_{1}$ имеет очень маленькую интенсивность. Результаты расчета спектральных характеристик РЕРС и его производных приведены в табл. 1.

Как видно из данных, оптически разрешенный переход на 336 nm для РЕРС, а также слабо разрешенные переходы около $360 \mathrm{~nm}$ для 2IPEРC и 3BrPEРC сформированы в основном перераспределением электронной плотности между орбиталями HOMO-LUMO с некоторой примесью соседних орбиталей. Как показал расчет, для мономера РЕРС основная часть электронной плотности НОМО-орбитали сконцентрирована вдоль длинной оси карбазольного цикла по связям между углеродными атомами (рис. 2). На орбитали НОМО-1 электронное облако сконцентрировано вдоль короткой оси карбазольного ядра и проходит по связям между атомами углерода. При

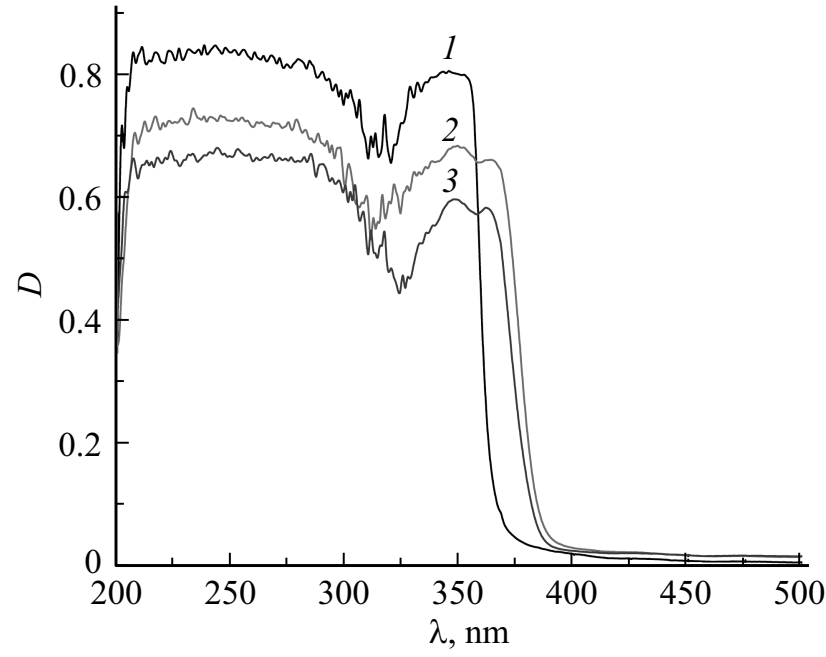

Рис. 3. Измеренные спектры поглощения пленок: 1 - РЕРС, 2 - 2IPEPC, 3 - BrPEPC.

возбуждении молекулы происходит концентрирование электронной плотности на атомах, имеющих большую величину заряда.

В присутствии тяжелых атомов наблюдаются небольшие изменения электронной плотности. В частности, атомы йода или брома перетягивают на себя часть электронной плотности в основном состоянии. При поглощении фотона происходит перераспределение электронной плотности по связям атомов углерода и атомами 

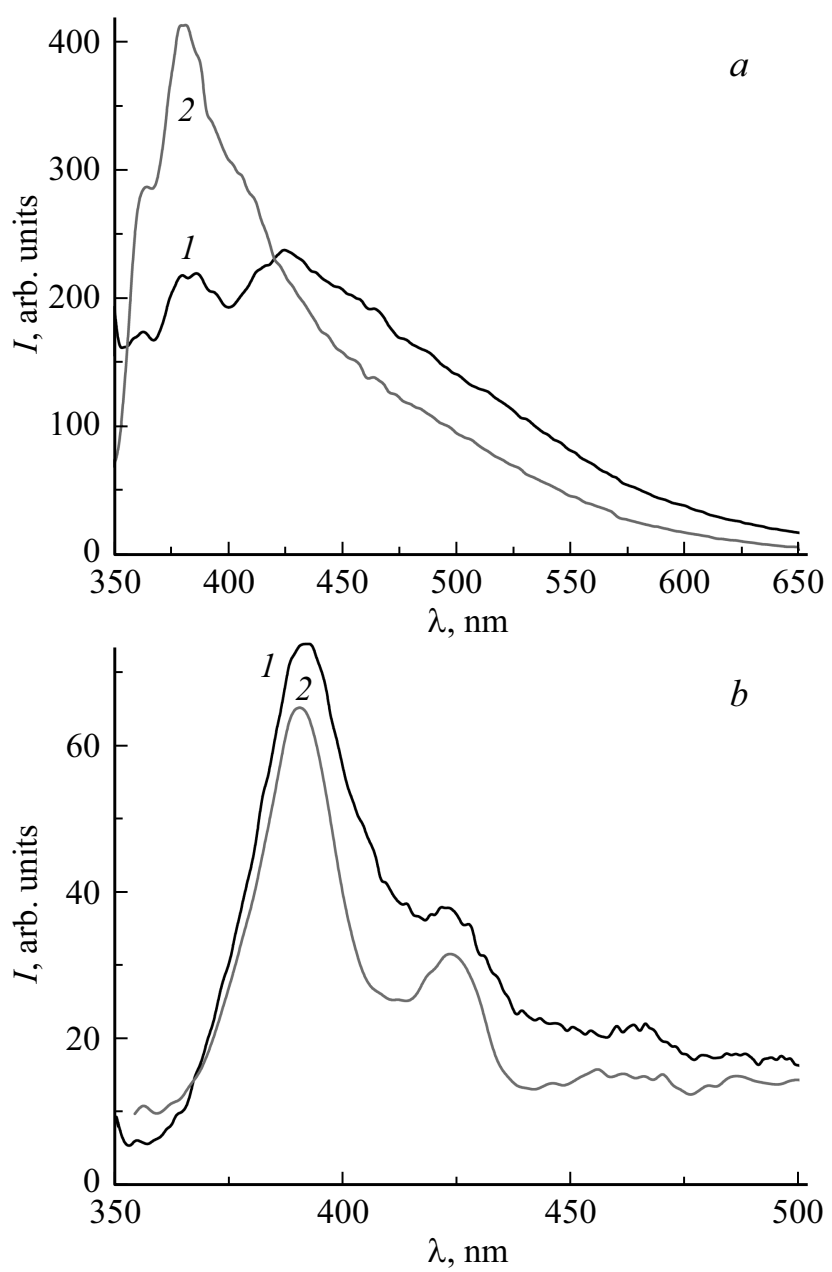

Рис. 4. Спектры флуоресценции пленок (a) РЕРС при различных температурах, $\lambda_{\mathrm{ex}}=340 \mathrm{~nm}: 1-293 \mathrm{~K}, 2-77 \mathrm{~K} ;(b)$ : $1-3$ BrPEPC, $2-2$ IРЕРС при $T=293 \mathrm{~K}$.

водорода, входящими в карбазольный остаток. При этом тяжелые атомы задействованы слабо.

Экспериментально полученные спектры поглощения полимерных пленок РЕРС и его производных представлены на рис. 3. Как видно из рисунка, спектры поглощения исследуемых полимеров расположены в виде широких полос в УФ области спектра. В спектре поглощения РЕРС можно выделить две полосы почти одинаковой интенсивности - в области $320-360 \mathrm{~nm} \mathrm{c}$ максимумом около $340 \mathrm{~nm}$ и полосой от 200 до $300 \mathrm{~nm}$. Край спектра поглощения приходится на $364 \mathrm{~nm}$. Спектр поглощения совпадает с данными, приведенными другими авторами[12-14]. В присутствии тяжелого атома наблюдается батохромный сдвиг длинноволновой полосы поглощения полимеров почти на $20 \mathrm{~nm}$ и проявление максимумов на 361 и $364 \mathrm{~nm}$. При этом поглощение в области 200-300 nm практически не изменяется.

При сравнении экспериментальных и рассчитанных спектров поглощения можно сказать, что коротковолновая полоса поглощения для всех трех полимеров на 200-300 nm сформирована в основном переходами с
Таблица 2. Времена жизни фосфоресценции полимерных пленок, $T=77 \mathrm{~K}$

\begin{tabular}{l|c|c}
\hline Образец & $\lambda_{\text {reg }}, \mathrm{nm}$ & $\tau, \mathrm{ms}$ \\
\hline 2ІРЕРС & 590 & 5 \\
3ВrРЕРC & 555 & 5 \\
РЕРС & 505 & 9
\end{tabular}

основного состояния $S_{0}$ на возбужденные состояния $S_{3}$ и выше. В их формировании преимущественно задействованы переходы между молекулярными орбиталями НОMO-1, НОMO-2, HOMO и LUMO+1. То есть поглощение в коротковолновой области связано с переходами из более глубоких молекулярных орбиталей карбазольной части РЕРС, энергия которых разнится на $0.3-0.4 \mathrm{eV}$ (табл. 1). Это формирует практически непрерывное поглощение [15]. Полосы поглощения в области 320-360 nm формируют переходы молекулы из $S_{0}$ в состояния $S_{1}$ и $S_{2}$ [16]. Как видно из данных табл. 1 и рис. 3 , данные переходы являются менее разрешенными и связаны с орбиталями HOMO-LUMO. Аналогичные данные были получены и при расчете мономеров 2IPЕРС и 3BrPEРС. Различия в силе осцилляторов переходов $S_{0} \rightarrow S_{1}, S_{0} \rightarrow S_{2}$ и $S_{0} \rightarrow S_{3}$ и выше наиболее заметны в случае добавления тяжелых атомов в структуру мономера, что видно из экспериментального спектра поглощения.

На основании расчетных и экспериментальных данных можно сделать вывод о том, что при добавлении тяжелого атома происходит длинноволновый сдвиг полос поглощения, что является следствием понижения энергии возбуждения из-за смещения распределения электронной плотности в системе $\pi$-электронов в хромофорах 2IPЕРС и 3ВrPEPC.

При исследовании спектрально-люминесцентных характеристик полимерных пленок РЕРС были получены спектры, показанные на рис. $4, a$.

Как видно из рисунка, в спектре флуоресценции пленок РЕРС наблюдается несколько пиков. Для пленки РЕРС при комнатной температуре основной максимум расположен на $426 \mathrm{~nm}$ с плечом около 380 и $364 \mathrm{~nm}$. Понижение температуры приводит к увеличению интенсивности свечения полимера на 364 и $380 \mathrm{~nm}$, а также к снижению интенсивности свечения на $426 \mathrm{~nm}$. Последняя полоса проявляется в виде плеча на кривой флуоресценции. Анализируя полученные данные, можно сказать, что излучение с максимумом на 360 и $380 \mathrm{~nm}$ является излучением мономерных центров, в то время как длинноволновая полоса излучения около $420 \mathrm{~nm}$ связана с образованием эксимеров [17]. Понижение температуры пленки приводит к снижению эффективности их образования, что является известным явлением [18]. В результате этого интенсивность мономерного свечения возрастает. 


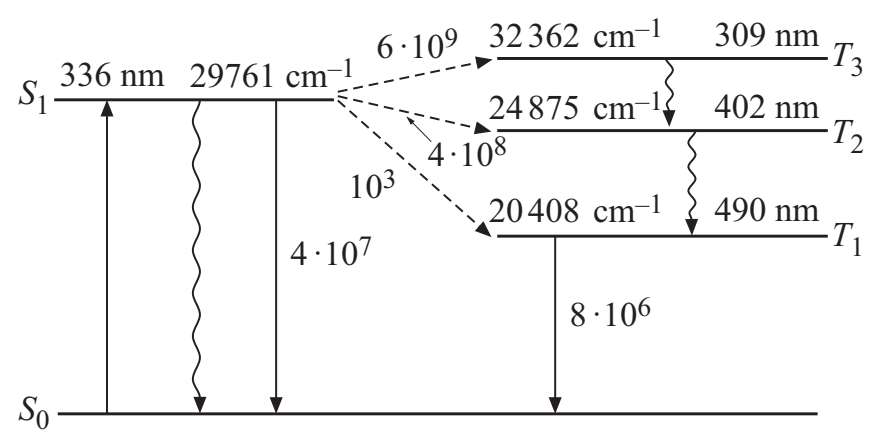

Рис. 5. Схема электронных состояний и внутримолекулярных переходов в мономерном звене РЕРС. Константы скоростей представлены в $\mathrm{s}^{-1}$.

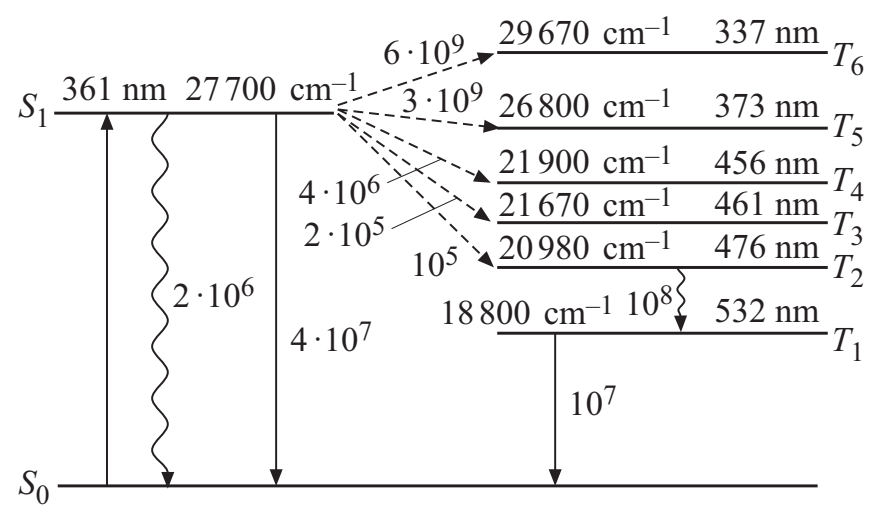

Рис. 6. Схема электронных состояний и внутримолекулярных переходов в мономерном звене 3BrPЕРС. Константы скоростей представлены в $\mathrm{s}^{-1}$.

Для полимеров с тяжелыми атомами наблюдается батохромный сдвиг полосы флуоресценции (рис. 4, $b$ ). При этом происходит изменение соотношений интенсивности свечения мономерных и эксимерных центров. Так, более интенсивным становится максимум на $390 \mathrm{~nm}$, а свечение около $422 \mathrm{~nm}$ проявляется в виде плеча спектра. При этом интенсивность флуоресценции пленок 3ВrPEPC и 2IPEPC почти в 3 раза меньше интенсивности свечения РЕРС. Времена жизни быстрой флуоресценции, зарегистрированные на $420 \mathrm{~nm}$, равны $4 \mathrm{~ns}$ для РЕРС, $1.1 \mathrm{~ns}$ для 2ІРЕРС и $1.6 \mathrm{~ns}$ для 3BrPEPC.

При исследовании влияния тяжелого атома на скорости фотофизических процессов в пределах мономерных звеньев РЕРС проводилась оценка констант скоростей излучательного процесса (сплошные линии), внутренней конверсии (волнистые линии) и интеркомбинационной конверсии (пунктирные линии). Для молекулы РЕРС была получена схема, показанная на рис. 5.

Из рисунка видно, что молекула, поглощая свет с $\lambda=340 \mathrm{~nm}$, переходит в состояние $S_{1}$. Далее она может либо перейти в состояние $S_{0}$, либо перейти в близлежащие триплетные состояния $T_{2}$ и $T_{3}$. Затем, посредством безызлучательной релаксации на уровень $T_{1}$, возможно испускание кванта фосфоресценции. Добав- ление тяжелого атома в структуру полимера приводит к понижению энергии триплетных состояний мономера (рис. 6). При этом увеличиваются также вероятность интеркомбинационных переходов из состояния $S_{1}$ и вероятность излучательного распада состояния $T_{1}$. Это значит, что в исследуемых полимерах весьма вероятно существование фосфоресценции. Для молекулы 2ІРЕРС рассчитанное положение триплетных уровней сдвигается незначительно в красную область (около $5 \mathrm{~nm}$ ), а скорости переходов имеют значения того же порядка, что и для 3BrPEPC.

Для подтверждения этого были измерены спектры фосфоресценции полимерных пленок (рис. 7,a). Спектр фосфоресценции РЕРС (кривая 1) представляет собой широкую полосу с максимумом на $490 \mathrm{~nm}$, которая сдвигается в длинноволновую область для макромолекул с тяжелыми атомами.

Максимум спектра фосфоресценции 3ВrPЕРС проявляется около $550 \mathrm{~nm}$, в то время как для 2ІРЕРС этот параметр приходится на $590 \mathrm{~nm}$. Зарегистрирован-
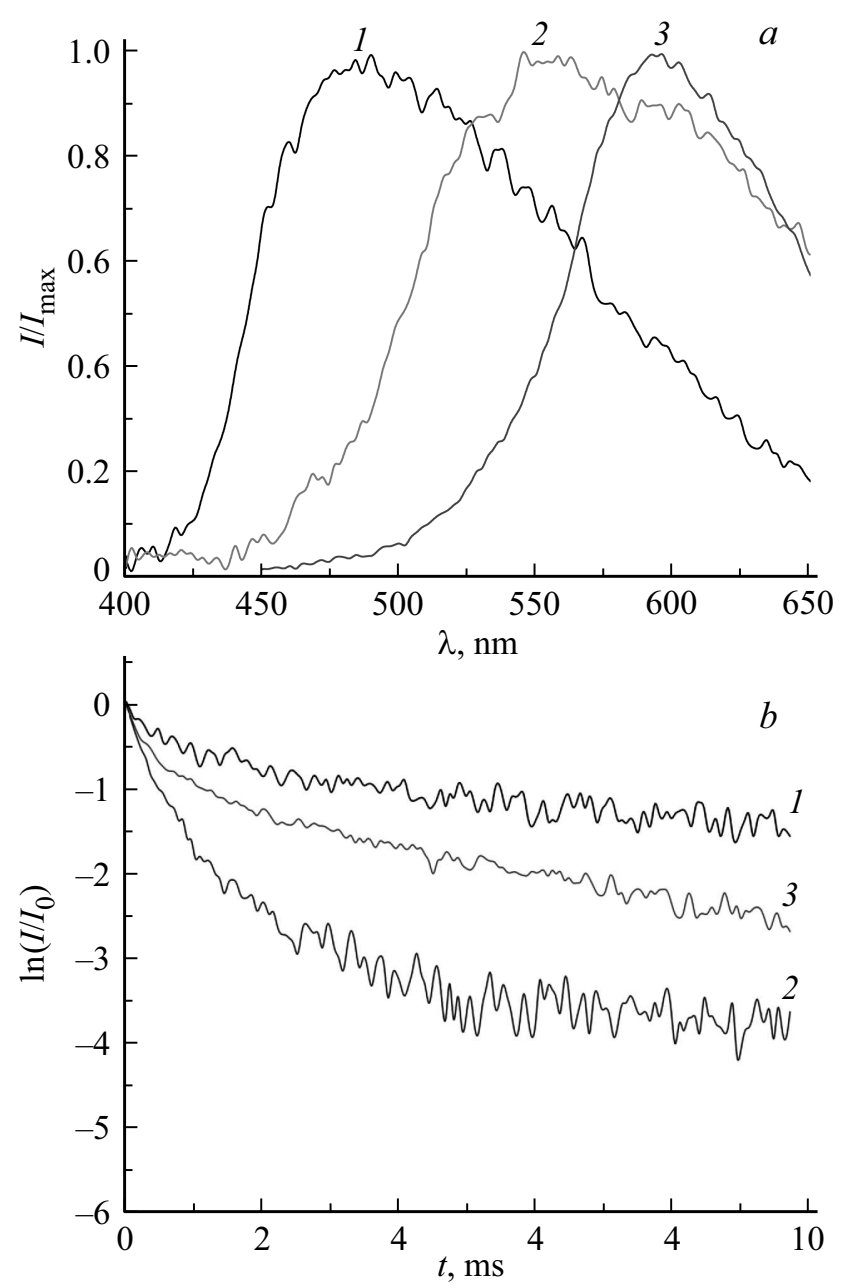

Рис. 7. (а) Нормированные спектры фосфоресценции пленок: 1 - РЕРC, $2-$ 2IPEPC, 3 - 3BrPEPC. $T=293 \mathrm{~K}$, $\lambda_{\mathrm{ex}}=340 \mathrm{~nm}$. (b) Кинетики фосфоресценции пленок: 1 PEPC, $2-$ 2IPEPC, $3-3$ BrPEPC. $T=77 \mathrm{~K}, \lambda_{\mathrm{ex}}=340 \mathrm{~nm}$. 
ные максимумы фосфоресценции близки по значению энергии с рассчитанным положением состояния $T_{1}$. При понижении температуры пленок до $77 \mathrm{~K}$ наблюдался рост интенсивности фосфоресценции без изменения положения полосы свечения. Кинетика фосфоресценции полимерных пленок показана на рис. 7,b. Времена жизни, вычисленные из долговременной части кривых затухания, приведены в табл. 2.

Как видно из рисунка, наличие тяжелого атома приводит к уменьшению времени жизни фосфоресценции. При

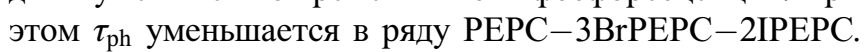
Следует отметить, что длительность свечения для всех образцов уменьшается с понижением температуры. С ростом величины тяжелого атома эта разница возрастает.

\section{Заключение}

Таким образом, при исследовании возбужденных синглетных состояний в фотопроводящих полимерах РЕРС и его производных с тяжелыми атомами установлено, что тяжелый атом в структуре полимерной молекулы приводит к батохромному сдвигу спектра поглощения. Это является следствием понижения энергии возбуждения из-за смещения распределения электронной плотности в системе $\pi$-электронов в хромофорах 2IPЕРС и 3ВrРЕРС. При этом коротковолновая полоса поглощения для всех трех полимеров на 200-300 nm сформирована в основном переходом между основным и высоковозбужденными состояниями $S_{3}$ и выше, в формировании которых преимущественно задействованы переходы между молекулярными орбиталями НОМО-1, НОМО и $\mathrm{LUMO}+1$. Полоса поглощения в области $320-360 \mathrm{~nm}$ связана с переходами между состояниями $S_{0} \rightarrow S_{1}$ и $S_{0} \rightarrow S_{2}$. При этом рассчитанная интенсивность длинноволновой полосы поглощения уменьшается в случае добавления тяжелых атомов в структуру мономера, что видно и в экспериментальном спектре поглощения.

Спектры люминесценции (как флуоресценции, так и фосфоресценции) пленок галогенсодержащих РЕРС также батохромно сдвинуты относительно РЕРС. При этом происходит изменение соотношений интенсивности свечения мономерных и эксимерных центров. Так, более интенсивным становится максимум на $390 \mathrm{~nm}$, а свечение около $422 \mathrm{~nm}$ проявляется в виде плеча спектра. Времена жизни флуоресценции в присутствии тяжелого атома также уменьшаются, что связано с увеличением вероятности интерконверсии в близлежащие триплетные состояния 3BrPEPC и 2IPEPC. Это было подтверждено при оценке величины констант фотофизических процессов. Показано, что для РЕРС высока вероятность как флуоресценции, так и фосфоресценции. При этом в галогенсодержащих РЕРС вероятность интеркомбинационной конверсии выше, что приводит к заметно более интенсивной фосфоресценции и сокращению ее времени жизни.
Настоящая работа выполнена в рамках научно-исследовательских грантов BR05236691 и АР05133724, финансируемых Министерством образования и науки Республики Казахстан.

Авторы выражают благодарность Н.А. Давиденко за предоставление полимеров.

\section{Список литературы}

[1] Арсланов В.В. // Усп. химии. 2000. Т. 69. № 10. С. 963.

[2] Давиденко И.И. Информационные среды. Киев: Киевский университет, 2010. 280 с.

[3] Zhao W., Qian D., Zhang Sh., Li S., Inganäs O., Gao F., Hou J. // Adv. Mat. 2016. V. 28. N 23. P. 4734. doi 10.1002/adma.201600281

[4] Singh-Rachford T.N., Castellano F.N. // Coordination Chem. Rev. 2010. V. 254. N 21-22. P. 2560. doi 10.1016/j.ccr.2010.01.003

[5] Keshtov M.L., Kuklin S.A., Khokhlov A.R., Konstantinov I.O., Nekrasova N.V., Xie Zhi-yuan, Biswasd S., Sharma G.D. /I New J. Chem. 2018. V. 42. P. 1626. doi 10.1039/C7NJ03981F.

[6] $\mathrm{Li}$ Zh., $X u \quad X$., Zhang $W$., Meng $X$., Genene Z., Ma W., Mammo W., Yartsev A., Andersson M.R., Janssen R.A.J. // Energy Environ. Sci. 2017. V. 10. P. 2212. doi 10.1039/C7EE01858D

[7] Ibrayev N.Kh., Afanasyev D.A., Ishcenko A.A., Davidenko N.A. // High Energy Chem. 2013. V. 47. N 2. P. 41. doi 10.1134/S0018143913020069

[8] Ibrayev N.Kh., Afanasyev D.A., Ishcenko A.A., Davidenko N.A. // Opt. Spectr. 2013. V. 114. N 1. P. 66. doi 10.1134/S0030400X13010128

[9] McGlynn S.P., Azumi T., Kinoshita M. Molecular Spectroscopy of the Triplet State. Prentice-Hall, 1969. 434 p.; Мак-Глинн С., Адзуми Т., Киносита М. Молекулярная спектроскопия триплетного состояния. М.: Мир, 1972. $448 \mathrm{c}$.

[10] Майер Г.В., Артюхов В.Я., Базыль О.К. Электронновозбужденные состояния и фотохимия органических соединений. Новосибирск: Наука, 1997. 213 с.

[11] Seliverstova E.V., Ibrayev N.Kh. // Opt. Spectrosc. 2017. V. 122. N 2. P. 207. doi 10.1134/S0030400X17020242

[12] Hari S.N. Advanced Functional Molecules \& Polymers: Electronic and Photonic Properties. Taylor and Francis group, 2001. $386 \mathrm{p}$.

[13] Wang Zi-Han, Lee H., Cu H.-N. // J. Appl. Phys. 2012. V. 111. P. 023512 . doi $10.1063 / 1.3678453$

[14] Bruno A., De Girolamo Del Mauro A., Nenna G. // J. Photonics for Energy. 2013. V. 3. P. 2. doi 10.1117/1.JPE.3.033599

[15] Pope M., Swenberg Ch. Electronic Processes in Organic Crystals and Polymers. N.Y.: Oxford University Press, 1999. 1360 р.; Поуп М., Свенберг Ч. Электронные процессы в органических кристаллах. М.: Мир, 1985. Т.2. 464 с.

[16] Скрышевский Ю.А. // ФТТ. 2010. Т. 52. № 6. С. 1227.

[17] Барашков Н.Н., Сахно Т.В., Нурмухаметов Р.Н., Хахель О.А. // Успехи химии. 1993. Т. 62 (6). С. 597. doi 10.1070/RC1993v062n06ABEH000032

[18] Гиллет Джс. Фотофизика и фотохимия полимеров. Введение в изучение фотопроцессов в макромолекулах. М.: Мир, 1988. 435 c. 